\title{
BLIND ADAPTIVE EQUALIZER FOR BROADBAND MIMO STBC BASED ON PDF FITTING
}

\author{
Samir Bendoukha, Adel Daas, and Stephan Weiss \\ Centre for Excellence in Signal \& Image Processing \\ Department of Electronic \& Electrical Engineering \\ University of Strathclyde, Glasgow, G1 1WX, Scotland, UK \\ \{samir, adel.daas, stephan\}@eee.strath.ac.uk
}

\begin{abstract}
In this paper, we propose a new blind adaptive technique used for the equalisation of space-time block coded (STBC) signals transmitted over a dispersive MIMO channel. The proposed approach is based on minimising the difference between the probability density function (PDF) of the equalizer output - estimated via the Parzen window method - and a desired PDF based on the source symbols. The cost function combines this PDF fitting with an orthogonality criterion derived from the STBC structure of the transmitted data in order to discourage the extraction of identical signals. This cost function motivates an effective and low-cost stochastic gradient descent algorithm for adapting the equaliser. The performance is demonstrated in a number of simulations and benchmarked against other blind schemes for the equalisation of STBC over broadband MIMO channels.
\end{abstract}

\section{INTRODUCTION}

The use of multiple transmit and receive antennas, commonly known as MIMO, has been shown to increase the capacity of a transmission link, [1]. This extra capacity can be exploited to increase the diversity gain of the system. The work in [2] proposed the STBC transmit diversity scheme, which is capable of maximising the diversity over frequency-flat MIMO channels, whose respsonses between pairs of transmit and receive antennas can be characterised by a complex gain factor.

For high data rate service, most channels cannot be considered frequency-flat anymore but are dispesrive, causing inter-symbol interference. In order to exploit diversity in such an environment, a number of variations on the classical STBC encoding have been proposed. OFDM can decompose a frequency-selective channel by introducing subcarriers and a cyclic prefix into a number of individual narrowband transmission channels, which can each be STBC encoded [3, 4]. The drawback of OFDM systems is in general the sensitivity to synchronisation errors and their large peak-to-average power ratio. Single-carrier time domain approaches were first proposed by [4], whereby the STBC structure was applied to a window of symbols, which is, after a guard interval, repeated as a complex conjugate and time reversed version [5]. However, time-reversal (TR) STBC is sensitive if the channel is doubly-dispersive, e.g. frequency selective and time-varying [6].

A recently proposed blind equalisation scheme for STBC [6] overcomes this problem and shows a higher robustness towards time-variations of the channel than TRSTBC. In order to increase the convergence speed of the STBC-CMA algorithm, various algorithmic variations have been evaluated recently, of which the most successful is the recursive quasi-Newton (RQN) approach, [7]. Unfortunately, the RQN method requires a considerable computational effort, which even a fast version of this algorithm cannot entirely aleviate.

In [8] a cost function that minimises the difference between a measured PDF at the equalizer output and a target PDF has been proposed, assuming a simple Gaussian model for the desired pdf. The technique has been successfully applied for SISO equalisation [8,9] and multiuser detection [10]. In this paper we intend to apply PDF fitting to target the equalisation of an STBC MIMO system. Based on the STBC-CMA approach [6], the aim of this paper is to investigate a PDF fitting based cost function for the equalisation of STBC over a broadband MIMO channel.

The paper is organised as follows. In Sec. 2 a brief description of the channel and signal model is given. The proposed cost function for the equaliser is detailed in Sec. 3, which motivates the derivation of a stochastic gradient algorithm in Sec. 4 in order to update the equaliser coefficients. Finally, Sec. 5 provides simulation results benchmarked against existing approaches to mitigate ISI in broadband MIMO STBC systems. Finally, conclusions are drawn in Sec. 6.

The following notations are used in this paper: $\mathscr{E}\{\cdot\}$ is the expectation operator. Vectors and matrices are denoted by bold face lower- and uppercase variables, e.g. $\mathbf{x}$ and $\mathbf{H}$, respectively, and $\{\cdot\}^{*},\{\cdot\}^{\mathrm{T}}$, and $\{\cdot\}^{\mathrm{H}}$ are the operators for complex conjugation, transposition and Hermitian transposition.

\section{CHANNEL AND SIGNAL MODEL}

The general setup of channel and equaliser is outlined in Fig. 1, and the individual system blocks are described below.

\subsection{Space-Time Block Coding}

Assuming transmission from two transmit antennas, the transmit signal $s[n]$ is STB encoded to provide two antenna signals $s_{i}[n], i \in\{1,2\}$ for the $i$ th antenna, computed according to [2],

$$
\left[\begin{array}{ll}
s_{1}[n] & s_{1}[n+1] \\
s_{2}[n] & s_{2}[n+1]
\end{array}\right]=\left[\begin{array}{lc}
s[n] & -s^{*}[n+1] \\
s[n+1] & s^{*}[n]
\end{array}\right]
$$

This produces two orthogonal signals with a characteristic STBC structure, which will later be exploited in the equaliser. 


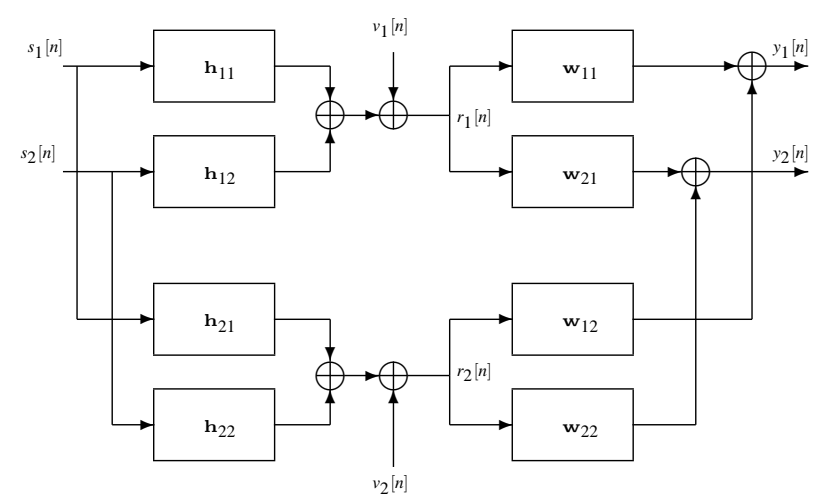

Figure 1: Channel and signal model with transmit STBC signals $s_{i}[n]$, a $2 \times 2$ MIMO channel with CIRs $h_{i j}[n]$, received signals $r_{j}[n]$, equaliser components $w_{j, i}[n]$ and equaliser outputs $y_{i}[n]$ which should adhere to the STBC structure.

\subsection{MIMO Channel Model}

The receiver requires a minimum of two receive antennas in order to equalise the MIMO system characterised above. Assuming the availability of two sufficiently spaced receive antennas, the signals $r_{j}[n], j \in\{1,2\}$, received at the $j$ th receive antenna over a dispersive and noise-corrupted channel are given by

$$
\left[\begin{array}{l}
r_{1}[n] \\
r_{2}[n]
\end{array}\right]=\sum_{v=0}^{N-1} \mathbf{H}_{n, v}\left[\begin{array}{l}
s_{1}[n-v] \\
s_{2}[n-v]
\end{array}\right]+\mathbf{v}_{n}
$$

The channel is characterised by the matrices

$$
\mathbf{H}_{n, v}=\left[\begin{array}{ll}
h_{1,1}[n, v] & h_{1,2}[n, v] \\
h_{2,1}[n, v] & h_{2,2}[n, v]
\end{array}\right],
$$

whereby $h_{j, i}[n, v]$ is the potentially time-varying channel impulse response between the $i$ th transmit and the $j$ th receive antenna, responding to an input $\delta[n-v]$. The vector

$$
\mathbf{v}_{n}=\left[\begin{array}{l}
v_{1}[n] \\
v_{2}[n]
\end{array}\right]
$$

represents spatially and temporally uncorrelated Gaussian noise with zero mean and covariance $\mathscr{E}\left\{\mathbf{v}[n] \mathbf{v}^{\mathrm{H}}[n]\right\}=\sigma_{v}^{2} \mathbf{I}$. The parameter $N$ in (2) is the length of the MIMO channel in (3) such that $\mathbf{H}_{n, v}=\mathbf{0} \forall n \geq N$.

\subsection{Equaliser}

For equalisation, we group the four subequalisers shown in Fig. 1 into two equalisers $\mathbf{w}_{i}, i \in\{1,2\}$, each responsible for the $i$ th output of the MIMO equaliser,

$$
\mathbf{w}_{i}=\left[\begin{array}{l}
\mathbf{w}_{i, 1} \\
\mathbf{w}_{i, 2}
\end{array}\right]
$$

with

$$
\mathbf{w}_{i, j}^{\mathrm{H}}=\left[w_{i, j}[0] \cdots w_{i, j}[L-1]\right], \quad\{i, j\} \in\{1,2\} .
$$

Similarly, the received samples from the $j$ th receive antenna sitting in the tap delay line of the subequaliser,

$$
\mathbf{r}_{j}^{\mathrm{T}}[n]=\left[r_{1}[n] \cdots r_{1}[n-L+1]\right],
$$

are concatenated into

$$
\mathbf{r}[n]=\left[\begin{array}{l}
\mathbf{r}_{1}[n] \\
\mathbf{r}_{2}[n]
\end{array}\right]
$$

With this notation, the outputs $y_{i}[n], i \in\{1,2\}$, of the equaliser in Fig. 1 over two successive symbol periods $n$ and $n+1$ can be written as

$$
\left[\begin{array}{ll}
y_{1}[n] & y_{1}[n+1] \\
y_{2}[n] & y_{2}[n+1]
\end{array}\right]=\left[\begin{array}{l}
\mathbf{w}_{1}^{\mathrm{H}} \\
\mathbf{w}_{2}^{\mathrm{H}}
\end{array}\right] \cdot[\mathbf{r}[n] \mathbf{r}[n+1]] .
$$

For successful equalisation, the equaliser output should match the channel input in an appropriate sense, and also reflect the STBC structure in (1). We will use these properties to construct a suitable cost function next.

\section{PDF-FITTING BASED COST FUNCTION}

\subsection{Cost Function Structure}

The proposed cost function is based on the idea of blindly equalising several signals, leading to a cost term which is here related to fitting the PDFs of the equaliser outputs to a desired PDF according to $[8,11]$. The danger of applying a blind criterion to every equaliser output $y_{i}[n]$ is that the strongest received signal will be extracted multiple times. In order to discourage multiple signal extraction, in [12] a cross-correlation criterion is added to the cost function. For STBC-CMA [6], this cross-correlation can be translated into an orthogonality condition $\xi_{\perp}$ of the two transmitted STBC signals. A similar approach is taken here, resulting in a combined cost function

$$
\xi=\alpha \sum_{i=1}^{N_{\mathrm{Tx}}} \xi_{\mathrm{PDF}, i}+(1-\alpha) \xi_{\perp}
$$

where $\xi_{\mathrm{PDF}, i}$ is the criterion to force the $i$ th output to attain a specific desired PDF, with $N_{\mathrm{Tx}}=2$ the number of transmitted symbols within the period of one STBC block. The parameter $0 \leq \alpha \leq 1$ controls the weighting between the two cost terms.

In the following, the PDF-fitting criterion for the extraction of signals at the equaliser outputs $y_{i}[n]$ is outlined in Sec. 3.2, followed by comments on the orthogonality condition to discourage multiple signal extraction in Sec. 3.3.

\subsection{PDF-Fitting Cost Function Component}

The idea of this approach is to measure the difference between two PDFs $p_{A}(z)$ and $p_{B}(z)$ [8],

$$
\xi_{\mathrm{PDF}}=\int_{-\infty}^{\infty}\left(p_{A}(z)-p_{B}(z)\right)^{2} d z
$$

Here, the variable whose PDF is measured at the equaliser output is the squared magnitude value $\left|y_{i}[n]\right|^{2}$, in close relation to the constant modulus algorithm. The estimation of this PDF is based on the Parzen window method, whereby a smooth PDF estimate is achieved by replacing a sample $\left|y_{i}[n]\right|^{2}$ by a kernel function centred at its location. We here select a Gaussian kernel $K_{\sigma}(z)$ with variance $\sigma$,

$$
K_{\sigma}(z)=\frac{1}{\sqrt{2 \pi} \sigma} e^{-\frac{z^{2}}{2 \sigma^{2}}},
$$


such that the PDF estimate over a window of $L$ output samples is given by

$$
\hat{p}_{\left|y_{i}[n]\right|^{2}}(z)=\frac{1}{L} \sum_{l=0}^{L-1} K_{\sigma}\left(z-\left|y_{i}[n-l]\right|^{2}\right) \quad .
$$

The larger the window length $L$, the more confident the PDF estimate will be. However, a trade-off exists, as during adaptation the output statistics are not stationary, therefore limiting $L$ to an interval within which the statistics can be assumed quasi-stationary.

The PDF estimate $\hat{p}_{\left|y_{i}[n]\right|^{2}}(z)$ will be compared to the PDF of the squared moduli of the transmitted signals, $\left|s_{i}[n]\right|^{2}$, subject to the same Gaussian kernel $G_{\sigma}(z)$ [9]. An advantage of convolving the discrete PDF of $\left|s_{i}[n]\right|^{2}$ defined by the constellation points of $s_{i}[n]$ with the kernel is that the resulting PDF exhibits a spread around constellation points akin to the influence of channel noise. Given the model of AWGN as outlined in Sec. 2.2, the PDF of squared moduli would be a superposition of chi-square distributions, which subsequently might provide a more appropriate kernel. However, we here follow the suggestion of a Gaussian kernel in [9] as this will lead to simplifications that are required for a solution with low computational cost.

Therefore, the convolution with the Gaussian kernel yields the desired PDF

$$
\hat{p}_{\left|s_{i}\right|^{2}}(z)=\frac{1}{M} \sum_{m=1}^{M} K_{\sigma}\left(z-\left|s_{i, m}\right|^{2}\right)
$$

where $s_{i, m}, m \in\{1,2, \cdots M\}$, are the $M$ constellation points of the $i^{t} h$ transmitted signal.

By expoiting the fact that for Gaussian kernels [9]

$$
\int_{-\infty}^{\infty} K_{\sigma}\left(z-z_{1}\right) K_{\sigma}\left(z-z_{2}\right) d z=K_{\sqrt{2} \sigma}\left(z_{1}-z_{2}\right)
$$

the matched-PDF component of the cost function for the $i$ th equaliser output simplifies for $L=1$ to, [8]

$$
\xi_{\mathrm{PDF}, i}[n]=-\frac{1}{M} \sum_{m=1}^{M} K_{\sqrt{2} \sigma}\left(\left|y_{i}[n]\right|^{2}-\left|s_{i, m}\right|^{2}\right) .
$$

The cost function component in (16) is depicted in Figure 2 in dependency of a single complex valued coefficient. Similar to the CMA, the cost function exhibits a manifold of optimum solutions due to its phase ambiguity.

\subsection{Orthogonality Condition}

Applying the PDF matching criterion of Sec. 3.2 to both equaliser outputs could potentially lead to the multiple extraction of only the strongest signal. Therefore, an additional constraint such as the minimisation of the cross-correlation between the equaliser outputs needs to be included into the cost function [12]. The orthogonality of the STBC structure provides a good condition which does not require the approximation of the cross-correlation over a window of data, but can be directly applied to data collected over two consecutive time slots [6].

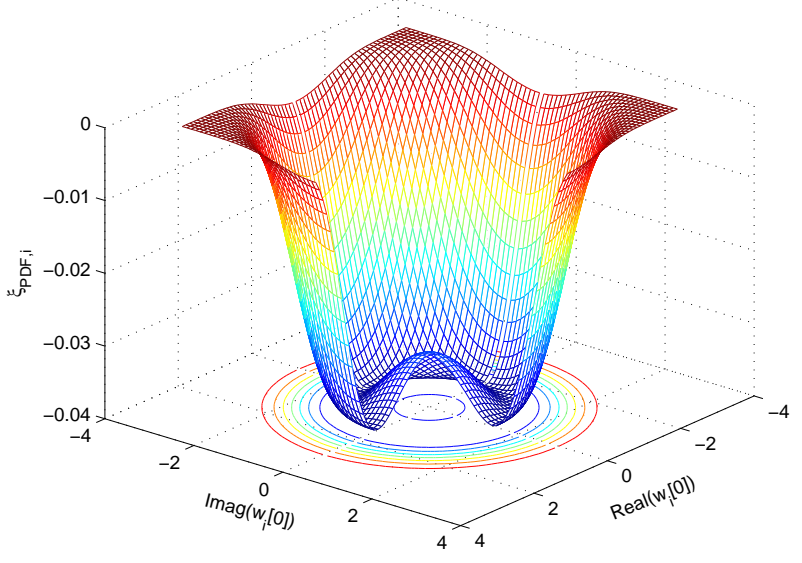

Figure 2: The PDF-matching cost function for one output. Part of the surface has been removed to visualise the shape near the origine.

Based on (1), in the absence of noise and for perfect equalisation we have $y_{1}[n]=y_{2}^{*}[n+1]=s[n]$ and $y_{2}[n]=$ $-y_{1}^{*}[n+1]=s[n+1]$. Thus the vector

$$
\mathbf{a}_{n}=\left[\begin{array}{lll}
y_{1}[n] & - & y_{2}^{*}[n+1] \\
y_{2}[n] & + & y_{1}^{*}[n+1]
\end{array}\right],
$$

provides a suitable measure of orthogonality, such that

$$
\xi_{\perp}=\mathbf{a}_{n}^{\mathrm{H}} \mathbf{a}_{n}
$$

If two orthogonal sequences are extracted, then the measure in (18) will be zero.

As the PDF-matching criterion is insensitive to the phase of the signals, the outputs $y_{i}[n]$ can be subject to an arbitrary rotation commonly found with constant modulus-type algorithms. If we introduce a rotation angle $\varphi_{i}$ for the $i$ th equaliser output

$$
\mathbf{a}_{n}=\left[\begin{array}{lll}
y_{1}[n] e^{j \varphi_{1}} & - & y_{2}^{*}[n+1] e^{-j \varphi_{2}} \\
y_{2}[n] e^{j \varphi_{2}} & + & y_{1}^{*}[n+1] e^{-j \varphi_{1}}
\end{array}\right],
$$

and assume perfect equalisation $y_{i}[n]=s_{i}[n]$, then

$$
\mathbf{a}_{n}^{\mathrm{H}} \mathbf{a}_{n}=0 \quad \Longleftrightarrow \quad \varphi_{1}=-\varphi_{2}+2 \pi k
$$

with $k \in \mathbb{Z}$. Thus, if the total cost function is minimised, the phase ambiguity of the PDF fitting cost function component is complemented by (20), enforcing the orthogonality component such that the equaliser outputs can be expected to match the transmitted signals save of opposite phase shifts.

\subsection{Overall Cost Function}

With the PDF fitting and orthogonality condition defined, the complete cost function outlined in (10) can be approximated as

$$
\begin{gathered}
\hat{\xi}_{n}=-\frac{\alpha}{M} \sum_{i=1}^{2} \sum_{v=0}^{1} \sum_{m=1}^{M} K_{\sqrt{2} \sigma}\left(\left|y_{i}[n+v]\right|^{2}-\left|s_{i, m}\right|^{2}\right)+ \\
+(1-\alpha) \mathbf{a}_{n}^{\mathrm{H}} \mathbf{a}_{n} .
\end{gathered}
$$


The PDF fitting component has an addition summation over two contributions, as the STBC code considered with (1) extends over two symbol periods.

\section{STOCHASTIC GRADIENT ALGORITHM}

Based on the cost function in (21), this section addresses the problem of adjusting the equaliser. We choose a stochastic gradient approach whereby updating occurs for every STBC block spanning two symbol periods. The coefficients of the $i^{t h}$ equaliser are updated using the stochastic gradient descent method,

$$
\mathbf{w}_{i}[n+2]=\mathbf{w}_{i}[n]-\mu_{P D F} \nabla_{\mathbf{w}_{i}^{*}} \hat{\xi}_{n},
$$

where $\nabla_{\mathbf{w}_{i}^{*}}$ denotes the gradient with regard to $\mathbf{w}_{i}^{*}$, and $\mu_{P D F}$ is the step size.

The PDF and the orthogonality enforcing components of the gradient $\hat{\xi}_{n}$ are derived separately below.

\subsection{PDF term}

The derivative of the PDF term with respect to $\mathbf{w}_{i}^{*}$ can be given by

$$
\frac{\partial}{\partial \mathbf{w}_{i}^{*}} \xi_{P D F \mid i}[n]=\frac{1}{M} \sum_{m=1}^{M} K_{\sqrt{2} \sigma}^{\prime}\left(\left|y_{i}[n]\right|^{2}-\left|s_{i, m}\right|^{2}\right) y_{i}^{*}[n] \mathbf{r}_{n},
$$

where

$$
K_{\sqrt{2} \sigma}^{\prime}(z)=\frac{\partial}{\partial z} K_{\sqrt{2} \sigma}(z)=-\frac{1}{4 \sigma^{2}} K_{\sqrt{2} \sigma}(z)
$$

is the derivative of the Gaussian kernel (12). In order to simplify the derivative, we assume all the points in the transmit constellation have the same modulus, i.e. $\left|s_{i, m}\right|^{2}=\gamma^{2}$, for $m=1, \cdots, M$. Thus, the summation and division by $M$ drops form (23). Evaluating the derivative $K_{\sigma}^{\prime}$ in (23) and rearranging terms leads to

$$
\frac{\partial}{\partial \mathbf{w}_{i}^{*}} \xi_{P D F \mid v}[n]= \begin{cases}K_{\sqrt{2} \sigma}\left(\left|y_{i}[n]\right|^{2}-\gamma^{2}\right) e^{*}[n] \mathbf{r}_{n} & v=i \\ \mathbf{0} & v \neq i,\end{cases}
$$

where,

$$
e^{*}[n]=\frac{1}{4 \sigma^{2}}\left(\left|y_{i}[n]\right|^{2}-\gamma^{2}\right) y_{i}^{*}[n],
$$

and $K_{\sqrt{2} \sigma}(z)$ is the Gaussial kernel used for the Parzen estimator as defined in (12) $[9,14]$. It is the additional of this kernel term that distinguishes this part of the cost function gradient shown in (25) from a standard CM algorithm.

\subsection{Orthogonality Condition}

Note that the second part of the cost function (10), $\xi_{\perp}$, is identical to the orthogonality-enforcing term of the STBCCMA cost function. The gradient of the term with regard to the space-time equalisers $\mathbf{w}_{i}, i \in\{1,2\}$, is given by

$$
\begin{aligned}
& \frac{\partial}{\partial \mathbf{w}_{1}^{*}} \xi_{\perp}=\left(y_{1}^{*}[n]-y_{2}[n+1]\right) \mathbf{r}_{n}+\left(y_{2}[n]+y_{1}^{*}[n+1]\right) \mathbf{r}_{n+1} \\
& \frac{\partial}{\partial \mathbf{w}_{2}^{*}} \xi_{\perp}=\left(y_{2}^{*}[n]+y_{1}[n+1]\right) \mathbf{r}_{n}+\left(y_{2}^{*}[n+1]-y_{1}[n]\right) \mathbf{r}_{n+1} .
\end{aligned}
$$

Inserting the combined terms (25) and (27) into (22) provides the update equation of the proposed algorithm. The constants arising e.g. from the differentiation of the kernel can be absorbed into the step size $\mu$.

\begin{tabular}{|c|c|c|}
\hline CMA & RQN-CMA & Matched-PDF \\
\hline \hline $24 L_{w}+4$ & $36 L_{w}^{2}+26 L_{w}+59$ & $24 L_{w}+6$ \\
\hline
\end{tabular}

Table 1: Complexity of the different equalisers, in number of Multiply-Accumulate (MAC) operations.

\begin{tabular}{|c|c|c|c|c|}
\hline delay & $T_{s}$ & $2 T_{s}$ & $3 T_{s}$ & $4 T_{s}$ \\
\hline \hline strength $[\mathrm{dB}]$ & 0 & -3 & -5 & -7 \\
\hline
\end{tabular}

Table 2: Delay-power profile of the MIMO system's channel impulse responses

\section{ALGORITHM PERFORMANCE}

This section provides details of the parameters of the algorithm and a comparison of its computational complexity, followed by simulations whose results are benchmarked against existing methods.

\subsection{Computational Complexity}

Tab. 1 lists the computational complexity of the proposed algorithm together with the STBC-CMA [6] and a fast converging, highly complex variant of the STBC-CMA based on a recursive Quasi-Newton update. The metric used here is to count complex multiply-accumulates (MAC). The difference between the proposed algorithm and the STBC-CMA is the evaluation of the Gaussian kernel in the update equation. The Gaussian kernel can be approximated by a Maclaurin series with only relatively few MACs but poor behaviour for larger arguments to the kernel. The best results we obtained by implement the Gaussian function as a look-up table which requires memory and comparisons, but only one multiplication.

\subsection{Channel Simulation and System Parameters}

A $2 \times 2$ MIMO model as indicated in Fig. 1 is used for simulations. It comprises of four dispersive channel impulse responses of length $L_{h}=4$ obeying the delay-power profile characterised in Tab. 2. Simulations are performed over an ensemble of 100 MIMO channels whose coefficients are drawn from complex Gaussian distributions with variances defined by Tab. 2.

QPSK modulation is used at the transmitter with a modulus equal to unity. At the receiver, signals are corrupted by AWGN at an SNR of $20 \mathrm{~dB}$.

The length of the subequalisers $\mathbf{w}_{i, j}, i, j \in\{1,2\}$ is set to $L_{w}=11$, and they are initialised to zero with only the middle tap of each subequaliser set to unity. The trade-off between the two cost function terms was selected as $\alpha=0.4$, and the step sizes for the various algorithms were selected such that they provided the approximately fastest yet still stable convergence across all channel realisations.

\subsection{Simulation Results}

Figure 3 shows the mean square error (MSE) curve of the proposed algorithm compared to those of the STBC-CMA and the RQN implementation at SNR $=20 \mathrm{~dB}$, whereby potential rotations as outlined in (20) have been compensated with respect to the steady-state performance. The results 


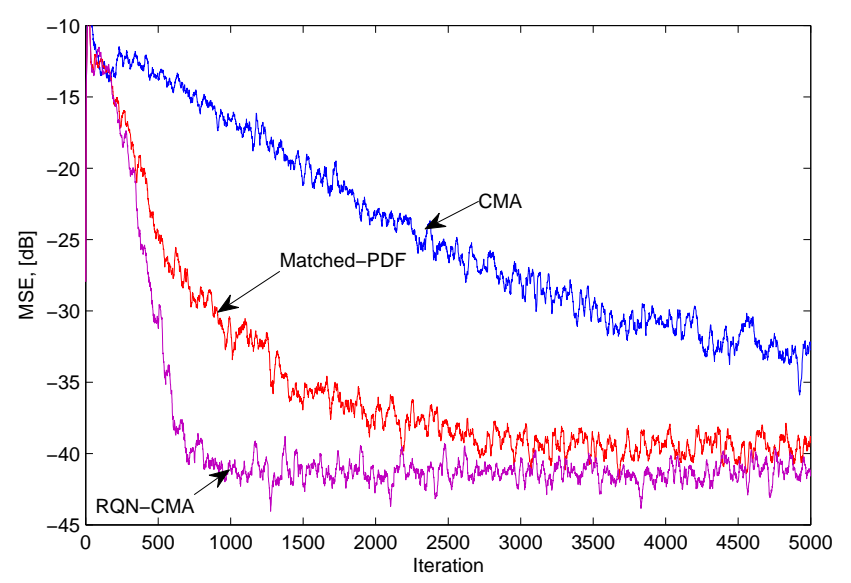

Figure 3: MSE curves of STBC-PDF and STBC-CMA.

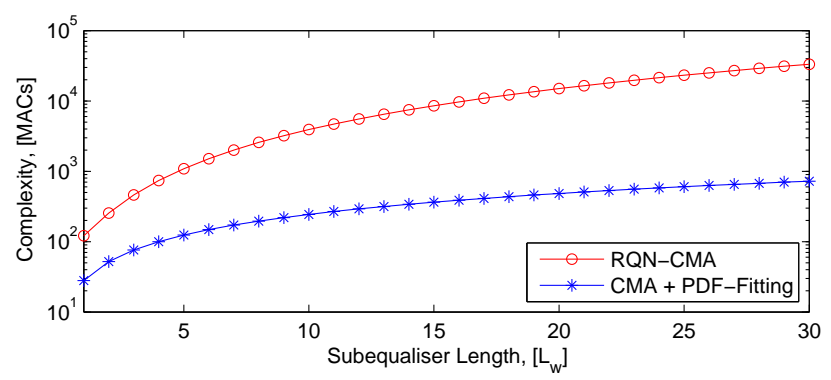

Figure 4: Complexity of the different equalisers, in number of Multiply-Accumulate (MAC) operations.

show that the MSE convergence of the PDF Matching Algorithm reaches the steady-state after fewer iterations compared to the STBC-CMA. The RQN equaliser shows the fastest convergence. However, as shown in Table 1, its complexity is of the order $\mathscr{O}\left(L_{w}^{2}\right)$ whereas the complexities of the STBC-CMA and the STBC-PDF schemes are of the order $\mathscr{O}\left(L_{w}\right)$. The complexity of the different equalisers is visualised in Fig. 4 as a function of the subequaliser length $L_{w}$. Both STBC-CMA and the proposed algorithm, have very similar computational cost.

\section{CONCLUSIONS}

A novel algorithm has been derived for the blind adaptive equalisation of STBC over frequency selective channels. The proposed cost function is based on minimizing the distance between the actual PDF of the equalizer output and a desired $\mathrm{PDF}$, and is complemented by an additional term that ensures the STBC structure and hence orthogonality of the equaliser outputs. A low-cost stochastic gradient update was derived based on this cost function.

The complexity of the derived algorithm was found to be comparable to the STBC-CMA, but the proposed method exhibited a faster convergence close to the performance of the fast but very costly recursive quasi-Newton version of the STBC-CMA.

\section{REFERENCES}

[1] G. J. Foschini and M. J. Gans. "On limits of wireless communication in a fading environment when us- ing multiple antennas.," in Wireless Personal Communications, , 6(3): 311335, March 1998.

[2] S.M Alamouti, "A simple transmit diversity technique for wireless communication," in IEEE Selected Areas in Communications., 16(8): 1451 1458, 1998.

[3] S. N. Diggavi, N. Al-Dhahir, A. Stamoulis, and A. R. Calderbank, "Differential space-time coding for frequency-selective channels," IEEE Communications Letters., 6(6): 253-255, June 2002.

[4] N. Al-Dhahir, "Overview and comparison of equalization schemes for space-time-coded signals with application to EDGE," IEEE Trans. on Signal Processing., 50(10): 2477-2488, Oct. 2002.

[5] R. Schober, H. Z. B. Chen, and W. H. Gerstacker. "Decision-feedback sequence estimation for timereversal space-time block-coded transmission," IEEE Trans. on Vehicular Technology, 53(4): 127321278, July 2004.

[6] S. Bendoukha and S. Weiss "Blind CM equalisation for STBC over multipath fading," IET ELECTRONICS LETTERS, 44(15): 922923, July 2008.

[7] S. Bendoukha and S. Weiss "A Fast Converging Blind Receiver for Space-Time Block Codes over Frequency Selective Channels," IEEE Int. Conf. on Signal Processing and Comms, 169-172, Nov. 2007.

[8] M. Lázaro, I. Santamaría, D. Erdogmus, K. Hild, C. Puntaleón, and J. C. Principe "Stochastic blind equalization based on PDF fitting using Parzen estimator," in IEEE Trans. on Signal Processing., 53: 696-704 , 2005.

[9] M. Lázaro, I. Santamaría, C. Puntaleón, D. Erdogmus, and J. C. Principe "Matched PDF-Based Blind Equalization," in IEEE International Conference on Acoustics, Speech, and Signal Processing ., 4: 297-300 , 2003.

[10] A. Daas, M. Hadef, and S. Weiss. "Adaptive blind multiuser equalizer based on pdf matching," In 16th Int. Conference on Telecommunications., Marrakech, Morocco, May 2009.

[11] E. Gokcay and J. C. Principe. "Information theoretic clustering," IEEE Trans. on Pattern Analysis and Machine Intelligence., 24(2):158-171, Feb. 2002.

[12] C. Papadias and A. Paulraj, "A Constant Modulus Algorithm for Multiuser Signal Separation in Presence of Delay Spread Using Antenna Arrays," IEEE SP Let., 4(6):178-181, 1997.

[13] C. B. Papadias. "Unsupervised receiver processing techniques for linear space-time equalization of wideband multiple input / multiple output channels," in IEEE Trans. on Signal Processing, 52(2): 472-482, , Feb 2004.

[14] S. Barbarossa and A. Scaglione, "Blind equalization using cost function matched to the signal constellation ," in Asilomar Conference on Signals, Systems \& Computers., 1: 550-554, 1997.

[15] J. Sala-Alvarez and G. Vazquez-Grau, "Stastistical reference criteria for adaptive signal processing in digital communications," in IEEE Trans. on Signal Processing., 45(1): 14 - 31, 1997. 